\title{
PROBLEMS FOR SOLUTION
}

P 125. Let $p$ be a prime $>5$. Show that there exist primes $q$ and $n$, both less than $p$, such that $q$ is a quadratic residue and $\mathrm{n}$ is a quadratic non-residue (mod $\mathrm{p}$ ).

J. Dixon, University of New South Wales

P 126. On any $\sigma$-finite, infinite measure space there exists a strictly positive, bounded function vanishing at infinity, but with infinite integral, and a similar function with finite integral.

J.E. Marsden, Princeton University

P 127. A spread in euclidean 3-space is a collection of skew lines with one line through every point. Give an easily visualized example.

J. Wilker, University of British Columbia

\section{SOLUTIONS}

P 110. Find the order, class, number of nodes, and number of cusps of the curve

$$
x_{1}^{2 / 3}+x_{2}^{2 / 3}+x_{3}^{2 / 3}=0
$$

in the complex projective plane.

H.S.M. Coxeter, University of Toronto

Solution by G. J. Griffith, University of Saskatchewan.

The curve $x^{2 / 3}+y^{2 / 3}+z^{2 / 3}=0$ is rational with parametric representation 


$$
\rho x=\left(1-t^{2}\right)^{3} ; \rho y=8 t^{3} ; \rho z=i\left(1+t^{2}\right)^{3} \text {. }
$$

Since it is clear that $x=\cos ^{3} \theta, y=\sin ^{3} \theta, z=i$ satisfies the equation. By letting $t=\tan ^{\theta} / 2$ we immediately obtain the above representation. Therefore the order of the curve is six.

The homogeneous line coordinates of the curve are

$$
\sigma u=2 t\left(1+t^{2}\right) ; \sigma v=1-t^{4} ; \sigma w=2 i t\left(1-t^{2}\right) \text {. }
$$

Therefore the order of the dual, and hence the class of the original curve is four. Since the curve is rational, it has genus zero.

The equation

$$
p=\frac{1}{2}(n-1)(n-2)-(\delta+k)
$$

[1] equation (3), page 100, and the so called first Plücker equation

$$
m=n(n-1)-2 \delta-3 k
$$

[1] equation (1), page 99, yield

$$
k=6 \text { and } \delta=4 \text {. }
$$

Equations (1') and (3) pages 99, 100 of [1] yield

$$
\imath=0 \text { and } \tau=3 \text {. }
$$

It is readily verified that the cusps are located at the points $(1, i, 0),(1,-i, 0),(1,0, i),(1,0,-i),(0,1, i)$ and $(0,1,-i)$ all lying on the conic $x^{2}+y^{2}+z^{2}=0$ and that the nodes are at the points

$$
(1,1,1),(1,1,-1),(1,-1,1)(-1,1,1)
$$

these real points being acnodes of the curve, whilst the bitangents are the sides of the triangle of reference having coordinates

$$
[1,0,0],[0,1,0],[0,0,1] \text {, }
$$


these being the cuspidal tangents.

It should be noted that this curve is projectively equivalent to the hypocycloid having four real cusps.

\section{REFERENCE}

1. Algebraic Plane Curves. J.L. Coolidge, Dover Publications, 1959. 


\section{$+$}

